\title{
Modal Decomposition of Operating Deflection Shapes Based on the General Definition of Projectors*
}

\author{
Takayuki KOIZUMI**, Nobutaka TSUJIUCHI** \\ and Yuichi MATSUMURA***
}

\begin{abstract}
This paper discusses the modal decomposition of the Operating Deflection Shapes (ODSs). The ODSs offer an important contribution in the understanding of the dynamic behavior of a system under operation. The modal decomposition can express each ODS as a linear combination of modes, and have been usually done in a least squares sense. However, there are scarcely modal vectors which cross at right angles, and consequently the results obtained from the ordinary method give obscure interpretation. Therefore we propose that the modal decomposition based on the General Definition of Projectors (GDOP) is most suitable for the detailed description of the modal decomposition. Moreover, we present a new model correlation technique based on the GDOP to verify the modal vectors, because the accuracy of the modal vectors is important in the modal decomposition. These approaches are demonstrated using numerically simulated FRFs and modal vectors. As a result, it was shown that these approaches give good clearer results compared to the ordinary modal decomposition method.
\end{abstract}

Key Words: Identification, Modal Analysis, Forced Vibration, Operating Deflection Shapes, Modal Decomposition

\section{Introduction}

In order to reduce a system's vibro-acoustical response observed under operating condition, the analysis of Operating Deflection Shapes (ODSs) ${ }^{(1)}$ plays an important role in the design of machines. To decompose the ODSs into underlying superposition of modal vectors has been studied to understand the contribution of each modes for the system's responses $^{(2)-(4)}$.

In particular, the modal decomposition of the

* Received 2nd November, 1998, Japanese original: Trans. Jpn. Soc. Mech. Eng., Vol.64, No.620, C (1998), p. 1170-1175 (Received 19th May, 1997)

** Department of Mechanical Engineering, Doshisha University, 1-3 Miyakodani, Tatara, Kyotanabe, Kyoto 610-0321, Japan. E-mail: tkoizumi@mail.do shisha.ac.jp

*** Department of Mechanical Systems Engineering, The University of Shiga Prefecture, 2500 Hassaka, Hikone, Shiga 522-8533, Japan
ODSs at a particular chosen frequency has been of great interest over the last few years. A peak in the spectra of the ODSs is mostly caused by a corresponding peak of the active forces rather than the resonance phenomenon in the analysis of mechanical structures equipped with engines such as automobiles, airplanes, and so on. Therefore, the ODSs for the chosen frequencies corresponding to engine speeds consist mainly of contribution of several modes associated to neighboring resonant frequencies. The analysis of the contribution is suggestive of the source of mechanical vibration.

In this paper, a modal decomposition based on General Definition of Projectors (GDOP) ${ }^{(5)}$ is discussed and compared to the ordinary modal decomposition ${ }^{(6)}$ based on Orthogonal Projection (OP) ${ }^{(7)}$. The GDOPbased modal decomposition gives detailed description of the contribution of each modes for the ODSs.

The GDOP assumes that a set of modal vectors is linearly independent. Therefore, it is important to check the linear independence relation among experi- 
mentally identified modal vectors in the pre-stage of modal decomposition. In general, the Modal Assurance Criteria $(\mathrm{MAC})^{(8)}$ is employed as correlation checks of modal vectors by many analysts, because the MAC requires no system matrices, such as mass or stiffness matrix. We, however, cannot precisely estimate the linear independence relation between mutually different modes with the MAC since the MAC is not a mass orthogonality check.

In order to assess the linear independence relation, it is necessary to calculate orthogonality using analytical system matrices ${ }^{(9)}$. In this calculation, model reduction is usually performed to reduce the system matrices to the set of experimental Degrees of Freedom (DOFs). However, the reduction of system matrices to the set of experimental DOFs is usually a difficult process. The second aim of the present work is to develop the orthogonality checks which can be computed without system matrices. We propose that the modal decomposition of experimental modal vectors by using the GDOP obtained from analytical modal vectors can be understood as an orthogonality check. This orthogonality check can be computed without Finite Element Analysis (FEA) mass matrix.

The computation of ordinary orthogonality checks is limited to real modal vectors. However, proposed checks can be performed with complex modal vectors. Because of this excellent feature, proposed checks can be done with experimental modal vectors.

This paper describes new modal decomposition and orthogonality checks. Then numerical example with lumped mass system is presented to show the effectiveness of the proposed two methods.

\section{Modal Decomposition}

Due to the major difficulty in knowing the operational forces, finite element model or experimentally estimated modal model usually does not offer the capability of simulating operational behavior. The remaining solution is the modal decomposition of ODSs measured under operating condition.

\subsection{Measurement of operating deflection shapes}

Operating responses may be measured simultaneously with the recent developments in multi-channel data acquisition system. However, there is surely the upper limit of channel count. The measurements have to be repeated for different sets of measurement locations.

In order to keep a fixed phase relation, a reference locations must be selected to which all responses are relatively measured. Let us assume that the number of measurement locations is $M$, and the reference location corresponds to the measurement loca- tion $q$. The crosspower spectrum is used for estimation of pseudo Frequency Response Functions (FRFs). The vector of the pseudo FRFs at a chosen frequency has same deflection patterns as ODSs. For the chosen frequency $\omega$, crosspower spectrum between the reference spectrum $y_{q}(\omega)$ and response spectrum $y_{i}(\omega), i=$ $1, \cdots, M$ is defined by

$$
G_{i q}(\omega)=y_{i}(\omega) \cdot y_{q}{ }^{*}(\omega)
$$

where $(\cdot)^{*}$ denotes the complex conjugate. Then vector of the ODS is given by

$$
\boldsymbol{x}(\omega)=\left[\begin{array}{c}
G_{1 q}(\omega) \\
G_{2 q}(\omega) \\
\vdots \\
G_{M q}(\omega)
\end{array}\right] .
$$

\section{2 Modal decomposition based on orthogonal projection}

Let us assume that the number of modes which are active in interested frequency range is $d$. We denote the modal vector sequence as $\Phi=\left[\boldsymbol{\phi}_{1}, \boldsymbol{\phi}_{2}, \cdots\right.$, $\left.\phi_{d}\right]$. The modal decomposition of the ODSs is given by

$$
x(\omega)=\eta_{1}(\omega) \phi_{1}+\eta_{2}(\omega) \phi_{2}+\cdots+\eta_{d}(\omega) \phi_{4}+\text { Rest }
$$

where Rest denotes a residual component, $\eta_{r}(\omega)$ is the modal weighting coefficient of a $r$ th mode, and this corresponds to the modal response amplitude in modal domain. This technique assumes that the system is proportionally damped.

Neyrinck et al. ${ }^{(6)}$ suggested that the modal decomposition can be done by using the orthogonal decomposition theorem ${ }^{(7)}$ :

$$
\eta_{r}=\left(\boldsymbol{\phi}_{r}^{T} \boldsymbol{\phi}_{r}\right)^{-1} \boldsymbol{\phi}_{r}^{T} \boldsymbol{x}(\omega)
$$

where $(\cdot)^{T}$ denotes transpose matrix.

Equation (4) means that the modal weighting coefficients is an orthogonal projection of $\boldsymbol{\phi}_{r}$ onto the subspace spanned by modal vector sequence $\boldsymbol{\Phi}$. However modal vectors usually do not span an orthogonal basis, and cosequently the modal weighting coefficients estimated by Eq. (4) has some error.

\section{3 Modal decomposition based on general definition of projectors}

Modal space is defined as the union of disjoint subspaces. Therefore, projection of ODSs onto the subspaces spanned by modal vectors can be defined by the GDOP(5) in detail.

We denote an $M$ dimensional vector space by $\boldsymbol{E}^{M}$ and a subspace spanned by the modal vector sequence $\boldsymbol{\Phi}$ by $S(\boldsymbol{\Phi})$. Let $\overline{\boldsymbol{\Phi}}_{r}$ be the matrix which contains modal vectors except for an $r$ th modal vector.

$$
\overline{\boldsymbol{\Phi}}_{r}=\boldsymbol{\phi}_{1} \cup \cdots \cup \boldsymbol{\phi}_{r-1} \cup \boldsymbol{\phi}_{r+1} \cup \cdots \cup \boldsymbol{\phi}_{d}
$$

We denote the direct sum of two disjoint subspaces $\boldsymbol{\phi}_{r}$ and $\boldsymbol{\phi}_{l}$ as $\boldsymbol{\phi}_{r} \oplus \boldsymbol{\phi}_{l}$.

If the Rest $=0$ and $\boldsymbol{E}^{M}=S\left(\boldsymbol{\phi}_{r}\right) \oplus S\left(\overline{\boldsymbol{\Phi}}_{r}\right)$, then 
projector $\boldsymbol{P}_{\phi_{r} \overline{\boldsymbol{\phi}}_{r}}$ which denotes the projector onto $S\left(\boldsymbol{\phi}_{r}\right)$ along $S\left(\overline{\boldsymbol{\Phi}}_{r}\right)$ can be defined as follows :

$$
\boldsymbol{P}_{\boldsymbol{\phi}_{r} \overline{\boldsymbol{\Phi}}_{r}}=\boldsymbol{\phi}_{r}\left(\boldsymbol{\phi}_{r}^{T} \boldsymbol{Q}_{\overline{\boldsymbol{\Phi}}_{r}} \boldsymbol{\phi}_{r}\right)^{\dagger} \boldsymbol{\phi}_{r}^{T} \boldsymbol{Q}_{\overline{\boldsymbol{\phi}}_{r}}
$$

where $\boldsymbol{Q}_{\overline{\boldsymbol{\Phi}}_{r}}=\boldsymbol{I}_{n}-\boldsymbol{P}_{\overline{\boldsymbol{\Phi}}_{r}}, \boldsymbol{P}_{\overline{\boldsymbol{\phi}}_{r}}$ denotes the orthogonal projector onto $S\left(\overline{\boldsymbol{\Phi}}_{r}\right)$ along the orthogonal subspaces of $S\left(\overline{\boldsymbol{\Phi}}_{r}\right)$ :

$$
\boldsymbol{P}_{\overline{\boldsymbol{\Phi}}_{r}}=\overline{\boldsymbol{\Phi}}_{r}\left(\overline{\boldsymbol{\Phi}}_{r}^{T} \overline{\boldsymbol{\Phi}}_{r}\right)^{-1} \overline{\boldsymbol{\Phi}}_{r}^{T}
$$

Then projection onto $r$ th mode along $S\left(\overline{\boldsymbol{\Phi}}_{r}\right)$ is calculated for Eq. ( 4$)$ :

$$
\boldsymbol{P}_{\boldsymbol{\phi}_{r} \overline{\boldsymbol{\Phi}}_{r}} \boldsymbol{x}(\omega)=\eta_{r}(\omega) \boldsymbol{\phi}_{r}
$$

Hence the modal weighting coefficient is obtained from

$$
\eta_{r}(\omega)=\boldsymbol{\phi}_{r}^{\dagger} \boldsymbol{P}_{\boldsymbol{\phi}_{r} \overline{\boldsymbol{\phi}}_{r}} \boldsymbol{x}(\omega) .
$$

If Rest $\neq 0$ and $\boldsymbol{E}^{M} \supset S\left(\boldsymbol{\phi}_{r}\right) \oplus S\left(\overline{\boldsymbol{\Phi}}_{r}\right)$, then projector $\boldsymbol{P}_{\phi_{r} \overline{\boldsymbol{\phi}}_{r}}^{\prime}$ which denotes the projector onto $S\left(\boldsymbol{\phi}_{r}\right)$ along $S\left(\overline{\boldsymbol{\Phi}}_{r}\right)$ can be defined as follows :

$$
\begin{aligned}
& \boldsymbol{P}_{\boldsymbol{\phi}_{r} \overline{\boldsymbol{\phi}}_{r}}^{\prime}=\boldsymbol{\phi}_{r}\left(\boldsymbol{\phi}_{r}^{T} \boldsymbol{Q}_{\overline{\boldsymbol{\phi}}_{r}} \boldsymbol{\phi}_{r}\right)^{\dagger} \boldsymbol{\phi}_{r}^{T} \boldsymbol{Q}_{\overline{\boldsymbol{\phi}}_{r}} \\
& \quad+\boldsymbol{Z}_{M}\left(\boldsymbol{I}_{M}-\boldsymbol{Q}_{\overline{\boldsymbol{\phi}}_{r}} \boldsymbol{\phi}_{r}\left(\boldsymbol{\phi}_{r}^{T} \boldsymbol{Q}_{\overline{\boldsymbol{\Phi}}_{r}} \boldsymbol{\phi}_{r}\right)^{\dagger} \boldsymbol{\phi}_{r}^{T}\right) \boldsymbol{Q}_{\overline{\boldsymbol{\phi}}_{r}}
\end{aligned}
$$

where $\boldsymbol{Z}_{M}$ is arbitrary $M$-dimensional square matrix. Hence the modal weighting coefficient is obtained from

$$
\eta_{r}(\omega)=\boldsymbol{\phi}_{r}^{\ddagger} \boldsymbol{P}_{\phi_{r}}^{\prime} \overline{\boldsymbol{\Phi}}_{r} \boldsymbol{x}(\omega) .
$$

\section{Correlation Checks of Modal Vectors}

To check the linear independence relation of modal vectors is indispensable to the GDOP-based modal decomposition. In this section, we introduce a new method for orthogonality checks, using the GDOP. This method is easier to use and saves remarkably efforts to perform orthogonality checks, because the method can be computed without using FEA mass matrix. In addition, this method can be applied even if the modal vectors are complex. Hence we can estimate the orthogonality checks among experimentally determined modal vectors.

In order to show the property of correlation checks, our orthogonality check is formulated as correlation checks between analytical and experimental modal vectors by way of intelligible and well known example.

At first, each of different correlation checks are briefly reviewed. The MAC is a well-known and widely used vector correlation check. Let analytical modal vectors be expressed as $\boldsymbol{\Phi}_{A}=\left[\boldsymbol{\phi}_{A 1}, \boldsymbol{\phi}_{A 2}, \cdots, \boldsymbol{\phi}_{A d}\right]$, and experimental modal vectors be expressed as $\boldsymbol{\Phi}_{X}=$ $\left[\boldsymbol{\phi}_{X 1}, \boldsymbol{\phi}_{X 2}, \cdots, \boldsymbol{\phi}_{X d}\right]$. The MAC is defined as

$$
\operatorname{MAC}\left(\boldsymbol{\phi}_{A k}, \boldsymbol{\phi}_{X l}\right)=\frac{\left|\boldsymbol{\phi}_{A k}^{T} \boldsymbol{\phi}_{X l}\right|}{\left\|\boldsymbol{\phi}_{A k}\right\|\left\|\boldsymbol{\phi}_{X l}\right\|}
$$

where $|(\cdot)|$ denotes absolute value and $\|(\cdot)\|$ denotes vector norm.

The MAC identifies the amount of vector correlation between $k$ th modal vector and $l$ th modal vector, and does not require an FEA mass matrix. The MAC can be used not only for normal modes, but also for complex modes. This method, however, cannot check the linear independence relation of modal vectors since the off-diagonal terms of MAC matrix only show the angle of modal vectors. The linear independence relation can be checked through well known mass orthogonality relation of normal modes. This can be done with the cross orthogonality checks (XOR)

$$
\mathrm{XOR}\left(\boldsymbol{\Phi}_{A}, \boldsymbol{\Phi}_{X}\right)=\boldsymbol{\Phi}_{A}^{T} \boldsymbol{M}_{A} \boldsymbol{\Phi}_{X}
$$

Here $\boldsymbol{M}_{A}$ denotes an analytical mass matrix. This method, however, cannot be used for complex modes, and also needs an analytical mass matrix.

To avoid these difficulties, we introduce a new formulation for orthogonality checks based on the GDOP. Concept for the orthogonality check via GDOP is summarized as the modal decomposition of modal vectors. Since subspaces spanned by FEA modal vectors are disjoint, the GDOP which is computed with the FEA modal vectors can maps EMA modal vectors onto each $\mathrm{FEA}$ modal vector along the rest of FEA modal vectors. Then we define the orthogonality check via GDOP as follows :

$$
\left[\begin{array}{c}
\left|\boldsymbol{\phi}_{A 1}^{\dagger} \boldsymbol{P}_{\phi_{A 1}} \overline{\boldsymbol{\Phi}}_{A 1} \boldsymbol{\Phi}_{X}\right| \\
\left|\boldsymbol{\phi}_{A 2}^{+} \boldsymbol{P}_{\phi_{A 2}} \overline{\boldsymbol{\Phi}}_{A 2} \boldsymbol{\Phi}_{X}\right| \\
\vdots \\
\left|\boldsymbol{\phi}_{A d}^{\dagger} \boldsymbol{P}_{\phi_{A d} \overline{\boldsymbol{\Phi}}_{A d}} \boldsymbol{\Phi}_{X}\right|
\end{array}\right]
$$

where $\boldsymbol{\Phi}_{A}$ and $\boldsymbol{\Phi}_{X}$ are normalized with vector norm. When experimental modal vectors $\boldsymbol{\Phi}_{X}$ are mapped into the subspace spanned by a $r$ th analytical modal vector $S\left(\phi_{A r}\right)$ along the subspace $S\left(\overline{\boldsymbol{\Phi}}_{A r}\right)$, the orthogonality check via GDOP computes the modal weighting coefficients of the $r$ th FEA modal vector. The orthogonality between these vectors would be indicated by all off-diagonal terms close to zero and by all diagonal terms close to one.

\section{Numerical Example}

In this section, we present some simulation results to illustrate the proposed two methods.

\subsection{Modal decomposition of FRFs}

Since each column of FRF matrix is superposition of modal vectors, and modal weighting coefficients can be exactly computed from FRF matrix, modal decomposition of FRF matrix is most suitable for

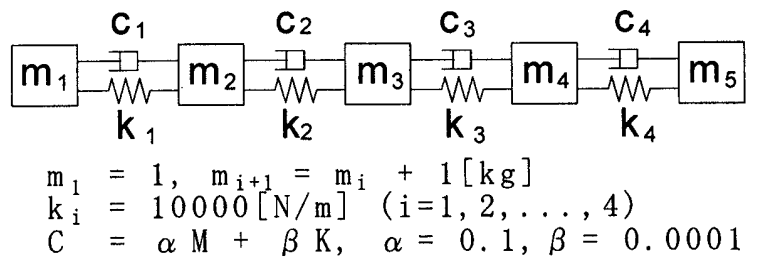

Fig. 1 5DOFs lumped mass model (free-free boundary condition) 


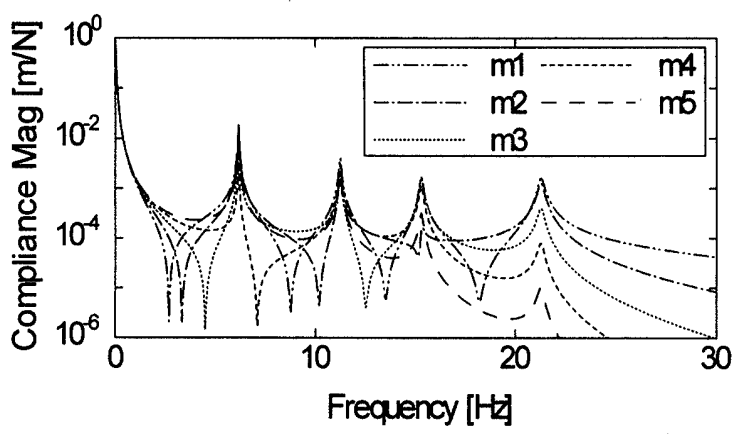

Fig. 2 Compliance FRF of 5DOFs lumped mass model

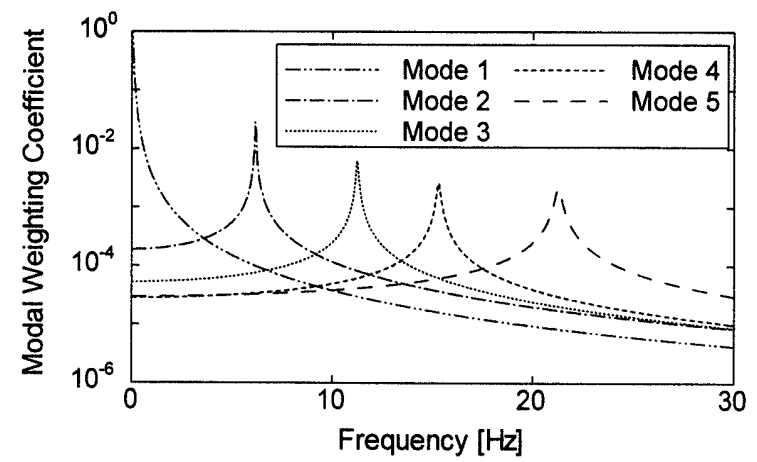

(a) Actual Modal Weighting Coefficient

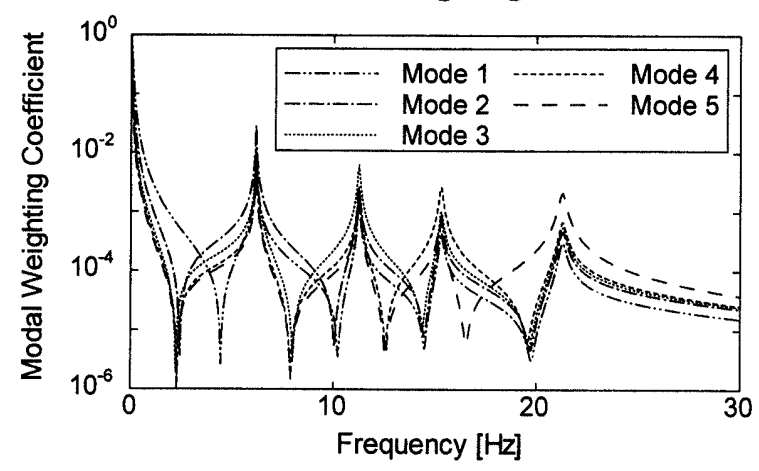

(b) Modal Weighting Coefficient Due to the OP

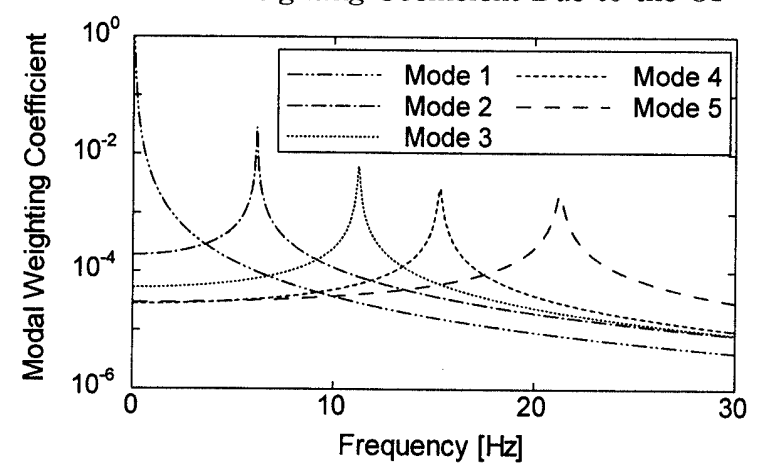

(c) Modal Weighting Coefficient Due to the GDOP

Fig. 3 Modal decomposition of FRF matrix (number of unknown modes $=0$ )

verifying the accuracy of modal decomposition. The modal decomposition of FRF matrix is herein used to compare the GDOP-based modal decomposition with the ordinary OP-based modal decomposition.

A 5DOFs lumped mass system shown in Fig. 1 was used to generate an FRF matrix. When the reference point is set to the 1st mass, the FRFs shown in Fig. 2 are computed for all of the measurement locations. The results of the modal decomposition for these FRFs are shown in Fig. 3. Figure 3 (a) shows actual value of modal weighting coefficients. Figure 3 (b) shows the results of OP-based modal decomposition. In this figure, the curves of identified modal weighting coefficients have five peaks. The reason why those have many peaks is that the orthogonal projection of modal vectors onto an any modal vector is influenced by the all modal vectors because modal vector sequence is not an orthogonal basis. Figure 3 (c) shows the results of GDOP-based modal decomposition. Identified modal weighting coefficients based on the GDOP have only one peak, and individual modal weighting coefficients are plotted in the FRF series as separate curves. This means that the formula for a projected modal vector based on the GDOP is exactly the same as the representation of the modal vector. This is useful feature for the modal decomposition because the modal weighting coefficients are the coefficients of modal vectors. Hence modal decomposition based on the GDOP is more accurate than ordinary one.

\section{2 Influence of unknown modal vector}

In the identification of ODSs, there is a case where unknown modes are excited. If the unknown modal components can be identified in the stage of modal decomposition, then we can investigate the cause of vibration. Therefore, we demonstrate the availability of GDOP-based modal decomposition for this case.

Let the 5th modal vector in the system shown in Fig. 1 be unknown. The results of modal decomposition based on $\boldsymbol{P}_{\phi_{r}, \overline{\boldsymbol{\phi}}_{r}}$ corresponding to the case Rest= 0 is shown in Fig. 4 (a), and the results of modal decomposition based on $\boldsymbol{P}_{\phi_{r} \overline{\boldsymbol{\phi}}_{r}}^{\prime}$ corresponding to the case Rest $\neq 0$ is shown in Fig. 4 ( b ). In Fig. 4 ( a ), the ODS of the unknown mode is approximately calculated as Rest because mapped components calculated as modal weighting coefficients of the unknown mode are smaller than the actual value shown in Fig. 3 (a). Figure 4 ( $\mathrm{b}$ ) shows that modal decomposition is done to reduce the Rest and to magnify the modal weighting coefficients around the 5th mode natural frequency. This feature lead us into difficulty for identifying the ODS corresponding to the 5th mode. Therefore, the GDOP corresponding to the case Rest $=0$ is suited to the modal decomposition of ODSs which include unknown modes.

\subsection{Modal decomposition with modal vector in error}

Experimentally determined modal vectors are 


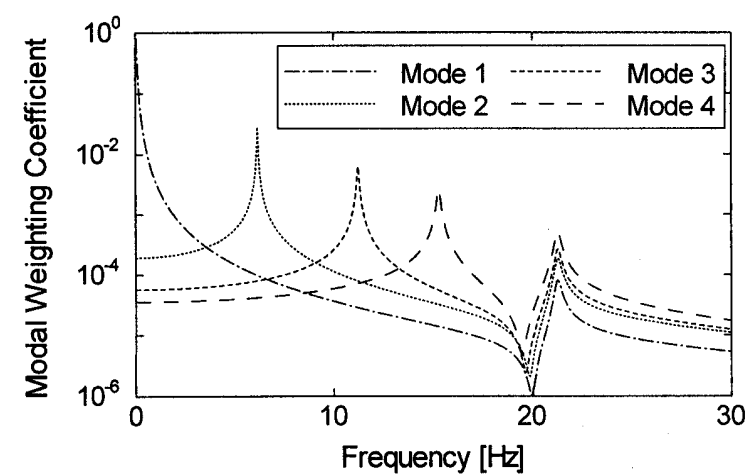

(a) GDOP (Assumption: $E^{M}=S\left(\phi_{i}\right) \oplus S\left(\bar{\Phi}_{i}\right)$ ).

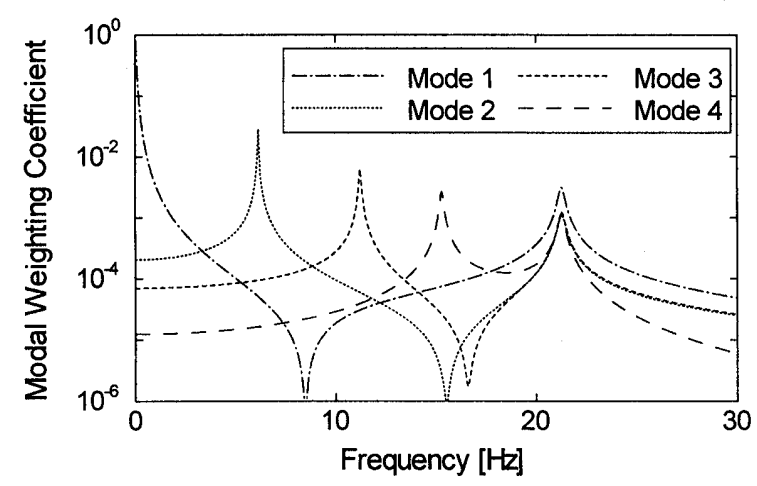

(b) GDOP (Assumption: $E^{M} \supset S\left(\phi_{i}\right) \oplus S\left(\bar{\Phi}_{i}\right)$ )

Fig. 4 Modal decomposition of FRF matrix (number of unknown modes $=1$ )

usually in error. The modal decomposition with modal vectors which are in error is herein used to compare the GDOP-based modal decomposition with the ordinary OP-based modal decomposition.

In this example, the system shown in Fig. 1 was modified. The weight of 1 st mass in the system was changed from $1.0[\mathrm{~kg}]$ to $1.5[\mathrm{~kg}]$. The modal vectors which are in error to perform modal decomposition were obtained from the modified system. The change of natural frequencies and the MAC value between changed modal vector and unchanged one are shown in Table 1. Modal decomposition was carried out using the FRFs shown in Fig. 2.

Results of the modal decomposition are shown in Fig. 5. The modal weighting coefficients estimated through the GDOP-based modal decomposition results in small error in comparison with those estimated through the OP-based modal decomposition. This tendency is notable in the low frequency range related to a rigid body mode. Since the rigid body mode is not changed by the system modification and measurement error, the GDOP-based modal decomposition which uses the rigid body mode in the every calculation have a clear advantage over the OP-based modal decomposition.

It is said that the elimination of the influence caused by rigid body modes is important in the ODS
Table 1 Change of natural properties

\begin{tabular}{c|c|c|c}
\hline \hline Mode & \multicolumn{2}{|c|}{ Natural Frequency } & MAC \\
\cline { 2 - 3 } No. & Before & After & Value \\
\hline 1 & 0.000 & 0.000 & 1.000 \\
2 & 6.182 & 5.906 & 0.998 \\
3 & 11.254 & 10.802 & 0.986 \\
4 & 15.311 & 14.794 & 0.970 \\
5 & 21.295 & 19.948 & 0.959 \\
\hline
\end{tabular}
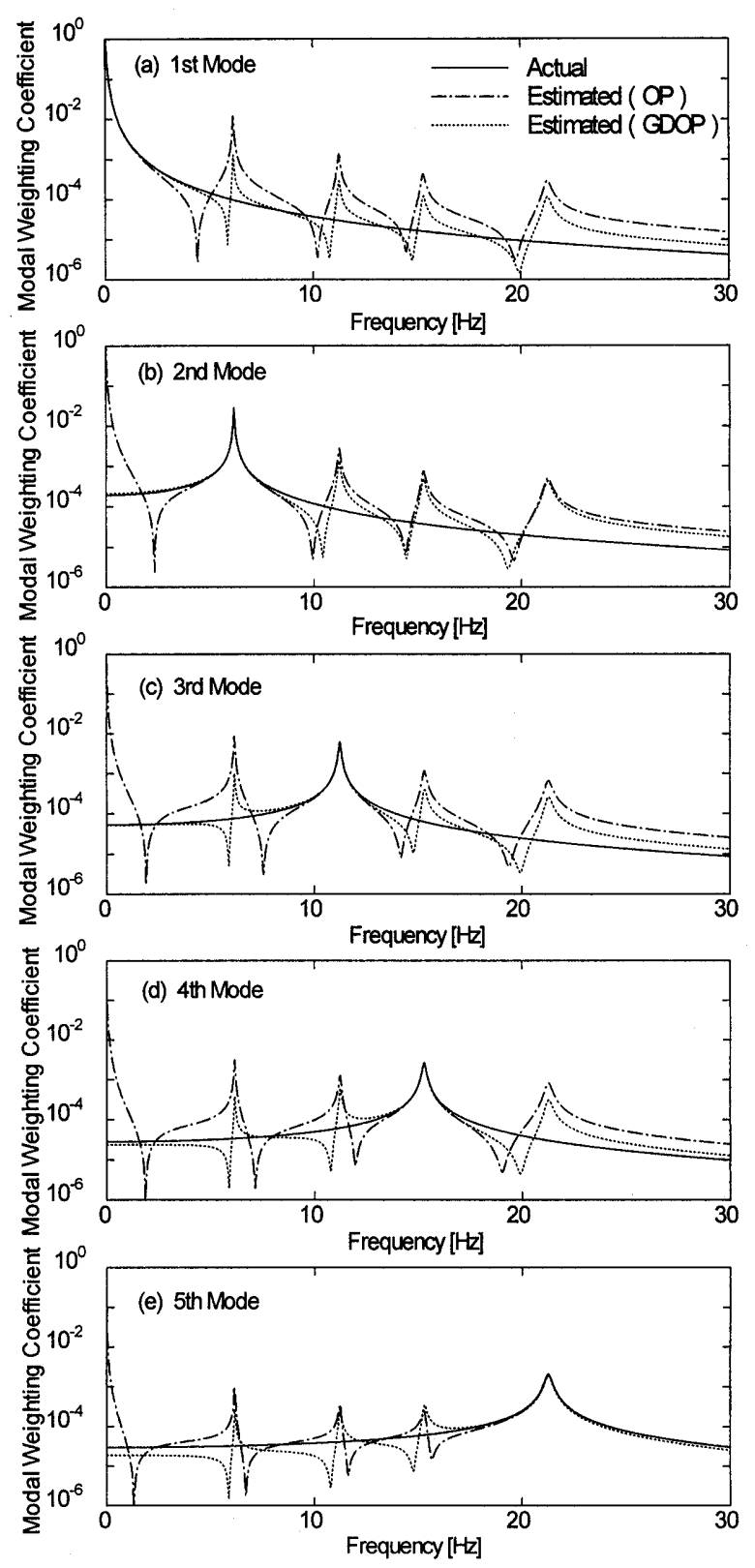

Fig. 5 Modal decomposition of FRF matrix with modal vectors which are in error

analysis of automobiles, because the car body generally has large ODSs caused by rigid body modes in the low frequency range. Therefore the GDOP-based modal decomposition is especially effective for this 
Table 2 Comparison of modal correlation method

\begin{tabular}{|c|c|c|c|c|c|c|}
\hline \multicolumn{7}{|c|}{ Modal Assurance Criteria } \\
\hline \multicolumn{2}{|c|}{ Mode No. } & 1 & 2 & 3 & 4 & 5 \\
\hline \multicolumn{2}{|c|}{ MAC Values } & 1.0001 & 1.000 & 0.991 & 1.000 & 1.000 \\
\hline \multicolumn{7}{|c|}{ Orthogonality Check } \\
\hline & & \multicolumn{5}{|c|}{ FEA } \\
\hline & Mode No. & 1 & 2 & 3 & 4 & 5 \\
\hline \multirow{5}{*}{ EMA } & 1 & 1.000 & 0.000 & 0.000 & 0.000 & 0.000 \\
\hline & 2 & 0.000 & 1.000 & 0.095 & 0.000 & 0.000 \\
\hline & 3 & 0.000 & 0.000 & 0.996 & 0.000 & 0.000 \\
\hline & 4 & 0.000 & 0.000 & 0.000 & 1.000 & 0.000 \\
\hline & 5 & 0.000 & 0.000 & 0.000 & 0.000 & 1.000 \\
\hline
\end{tabular}

\begin{tabular}{l|c|ccccc}
\hline \multicolumn{7}{c}{$\begin{array}{c}\text { Orthogonality Check Based on } \\
\text { General Definition of Projectors }\end{array}$} \\
\hline \hline & & \multicolumn{5}{c}{ FEA } \\
\hline & Mode No. & 1 & 2 & 3 & 4 & 5 \\
\hline \multirow{6}{*}{ EMA } & 1 & 1.000 & 0.000 & 0.000 & 0.000 & 0.000 \\
& 2 & 0.000 & 1.000 & $\mathbf{0 . 1 0 4}$ & 0.000 & 0.000 \\
& 3 & 0.000 & 0.000 & $\mathbf{1 . 0 3 9}$ & 0.000 & 0.000 \\
& 4 & 0.000 & 0.000 & 0.000 & 1.000 & 0.000 \\
& 5 & 0.000 & 0.000 & 0.000 & 0.000 & 1.000 \\
\hline
\end{tabular}

kind of ODS analysis.

4. 4 Comparison of modal correlation techniques

A simulation result is presented to illustrate the adequacy of proposed orthogonality checks based on GDOP. The system shown in Fig. 1 was used for computing of modal vectors $\Phi=\left[\boldsymbol{\phi}_{1}, \boldsymbol{\phi}_{2}, \boldsymbol{\phi}_{3}, \boldsymbol{\phi}_{4}, \boldsymbol{\phi}_{5}\right]$. In order to make the role of off-diagonal elements in the orthogonality check matrix clear, we treat a special case: when there are modal coupling in measured FRFs, there is a strong possibility that the EMA modal vectors estimated from coupling modes are influenced one another.

In this example, we set actual modal vectors of the system as the FEA and EMA modal vectors except for a 3rd EMA modal vector.

$$
\begin{aligned}
& \boldsymbol{\Phi}_{A}=\left[\boldsymbol{\phi}_{1}, \boldsymbol{\phi}_{2}, \boldsymbol{\phi}_{3}, \boldsymbol{\phi}_{4}, \boldsymbol{\phi}_{5}\right] \\
& \boldsymbol{\Phi}_{X}=\left[\boldsymbol{\phi}_{1}, \boldsymbol{\phi}_{2}, \boldsymbol{\phi}_{3}+0.1 \boldsymbol{\phi}_{2}, \boldsymbol{\phi}_{4}, \boldsymbol{\phi}_{5}\right]
\end{aligned}
$$

The modal vectors used in the calculation were normalized to unity vector. The comparison of modal correlation techniques were made with respect to the full model. The results are shown in Table 2.

The off-diagonal terms of MAC matrix are not mentioned in the Table 2, because those are essentially not zeros and accordingly those do not reveal the linear independence relation of modal vectors. In the both orthogonality checks, influence of the 2nd mode on the 3rd mode is denoted by both the offdiagonal term $(2,3)$ and the diagonal term $(3,3)$.

When $\boldsymbol{\Phi}_{X}$ is mapped into $S\left(\boldsymbol{\phi}_{A 2}\right)$ along $S\left(\overline{\boldsymbol{\Phi}}_{A 3}\right)$, the component $0.1 \phi_{2}$ in $\boldsymbol{\phi}_{X 3}$ appears on the off-diagonal term $(2,3)$. On the other hand, when $\boldsymbol{\Phi}_{X}$ is mapped into $S\left(\boldsymbol{\phi}_{A 3}\right)$ along $S\left(\overline{\boldsymbol{\Phi}}_{A 3}\right)$, the component $0.1 \boldsymbol{\phi}_{2}$ in $\boldsymbol{\phi}_{X 3}$ changes the diagonal term $(3,3)$ into a figure except unity. The calculation of the orthogonality check via GDOP can be done without FEA mass matrix, and accordingly this technique is easier to use than ordinary one.

\section{Concluding Remarks}

This paper described a new method for both modal decomposition and a orthogonality check based on the general definition of projectors. Then these techniques applied to the simulated frequency response functions and modal vectors, and the followings were observed.

1. The modal decomposition based on the general definition of projectors is theoretically more proper than ordinary modal decomposition based on the orthogonal projection.

2. When the operating deflection shapes have the influences of unknown modes, the modal decomposition based on the general definition of projection which is defined for the case that all modal vectors are known is effective for the operating deflection shapes analysis in order to make the components of unknown modes clear.

3. When the operating deflection shapes have the influences of rigid body modes, the modal decomposition based on the general definition of projectors is especially effective, because the rigid body mode is not changed by the system modification and measurement error, the modal decomposition based on the general definition of projectors which uses the rigid body mode in the every calculation have a clear advantage over the ordinary modal decomposition based on the orthogonal projection.

4. The calculation of the orthogonality checks via the general definition of projection can be done without FEA mass matrix, and also the results of the calculation are similar to those of ordinary orthogonality checks.

\section{References}

(1) Richardson, M.H. and McHargue, P.L., Operating Deflection Shapes from Time Versus Frequency Domain Measurements, Proc. of the 12th IMAC, (1994), p. 581-587.

(2) Matsumura, Y., Koizumi, T. and Tsujiuchi, N., Modal Parameter Identification Using the Concept of ESPRIT, Proc. of the 14th IMAC, (1996), p. 682-688.

(3) Matsumura, Y., Tsujiuchi, N. and Koizumi, T., Fast Identification Method of Operating Deflection Shapes, Proc. of the 3rd Inter. Conf. on MOVIC, (1996), p. 502-507.

(4) Tsujiuchi, N., Koizumi, T. and Matsumura, Y., Analysis of Operating Deflection Shapes Based on 
Subspace Method, Proc. of ASME DETC '97, (1997).

(5) Rao, C.R. and Yanai, H., General Definition and Decomposition of Projectors and Some Applications to Statistical Problems, J. of Statistical Planning and Inference, Vol. 3 (1979), p. 1-17.

(6) Neyrinck, F., et al., Operational Analysis and Problem Identification of a High Performance Racing Engine, Proc. of the 10th IMAC, (1992), p. 1399-1405.

(7) Lay, D.C., Linear Algebra and Its Application,
(1994), p. 352, Addison-Wesley Publishing Company.

(8) Allemang, R.J. and Brown, D.L., A Correlation Coefficient for Modal Vector Analysis, Proc. of the 1st IMAC, (1982), p. 110-116.

(9) Avitabile, P., Pechinsky, F. and O' Callahan, J.C., Study of Modal Vector Correlation using Various Techniques for Model Reduction, Proc. of the 10th IMAC, (1992), p. 572-583. 